\title{
STRINGS IN PLANE WAVE BACKGROUNDS REVISITED
}

O. Jofre and C. Núñez

Instituto de Astronomía y Física del Espacio

C.C. 67, Suc. 28, 1428 Buenos Aires,

Consejo Nacional de Investigaciones Científicas y Técnicas, Argentina.

\begin{abstract}
String theory in an exact plane wave background is explored. A new example of singularity in the sense of string theory for nonsingular spacetime metric is presented. The 4-tachyon scattering amplitude is constructed. The spectrum of states found from the poles in the factorization turns out to be equivalent to that of the theory in flat space-time. The massless vertex operator is obtained from the residue of the first order pole.
\end{abstract}

\section{Introduction}

As a fundamental generalization of Einstein gravity and Yang-Mills theory, the concepts behind string theory remain quite mysterious. Although non-perturbative effects are being slowly discovered, at the moment we only have a perturbative formulation of a yet unknown full quantum theory. In the present framework, gravity appears at different levels. The string spectrum in flat Minkowski space-time contains gravitons whose interactions determine the classical backgrounds consistent with the string dynamics. The appearance of a non-trivial curved geometry as an infinite genus effect was realized by Amati and Klimcik 4 . They showed the coincidence between the summation of string loop diagrams of high energy graviton scattering in flat space-time performed in [2] with the S-matrix relating in and out free excitations of a string in a shock wave background metric. The interplay between the different hierarchies assumed by gravity in these schemes is still unclear.

In the absence of an adequate understanding of the string picture, a classification of the specific symmetries is a useful tool in the search of the definite theory. The possible geometries compatible with compactification to 4 dimensions provide information about these symmetries (for instance the classification of acceptable string vacua has made manifest the existence of mirror symmetry) and allows identification of phenomenologically viable models. A deeper insight has been gained by formulating the theory in background fields. Duality symmetry, first discovered in toroidal

compactification 3] was generalized to any non-linear $\sigma$-model with an isometry in [4 and is considered a potential string answer to the occurrence of singularities and the problem of the cosmological constant in general relativity 5 . Many interesting string backgrounds have been identified starting from the WZW model. The requirement that the theory be conformal invariant at the quantum level amounts to the vanishing of the $\beta$-functions of the couplings of the nonlinear $\sigma$-model. WZW models are exactly splvable CFT and thus exact solutions to these equations. Gauging a one dimensional subgroup of SL(2R) Witten 16 found an exact 2D black hole. The 3D black hole background was recently obtained in ref. [5] The discrete symmetries allow to identify mathematically equivalent vacua with radically different geometries. The 2 and 3D black holes are examples of conformal field theories with two space-time interpretations related by duality.

The computation of scattering amplitudes is the main physical task in string theory which is basically an S-matrix theory. Particles of various masses and spins are exchanged in the different channels of a scattering process. They appear as poles in the square momentum when the points where some of the external vertices are attaghed coincide. However when the theory is formulated in background fields other (genuine) divergences could appear 6.6 in physical operators and in the partition function. Furthermore, the spectrum of string states can be modified as compared with the flat space theory 8$]$.

Our aim in this paper is to further explore the consequences of string theory in nontrivial backgrounds. We reconsider the bosonic string in exact plane wave metrics. In section 2 we review reference [7] and present new exact results for the string mass operator in certain shapes of the wave. In section 3 we consider the scattering amplitude of tachyons in this background. The spectrum of states obtained from the poles of the amplitude upon factorization is shown to be equivalent to that of the flat space-time theory. From Taylor expanding this residue the vertex operators of the corresponding exchanged states can be extracted. Indeed, the residue corresponds to the scattering amplitude of the intermediate higher mass states with the remaining tachyons. This is done in section 4 where the massless vertex operator is obtained. Final conclusions and discussion of the results are contained in section 5 .

Similar issues have been recently addressed in reference 13 from the current algebra giving rise to certain gravitational waves different from those considered here.

\section{Strings in plane wave backgrounds}

In order to establish notation and to be self-contained, this section summarizes some of the results of reference [7] and at the end we present new results.

The so called plane fronted waves in D dimensions:

$$
d s^{2}=-d U d V+d X^{a} d X_{a}+F\left(U, X^{a}\right) d U^{2}
$$

with $U=T-X^{D}, V=T+X^{D}$ and $X^{a}$, a=1,2,.., D-2 transverse coordinates, are solutions to all orders of the conformal invariance conditions of the bosonic string theory (the $\beta$-functions of the non-linear $\sigma$-model) in an $\alpha^{\prime}$ 
expansion and exen-non-perturbatively 14 . String theory in gravitational waves has been extensively discussed in the literatture 1 6.64. A monochromatic plane wave background was recently constructed by Nappi and Witten 15$]$ from an ungauged WZW model based on a central extension of the 2D Poincaré algebra. The conformal field theory description of such algebra and its cosets was considered in ref. 13. Plane waves are interesting because the existence of a covariantly constant null vector leads to a definition of frequency which is conserved, and therefore there is no analogue of the particle creation mechanism of semiclassical theories in curved space-times. This is not true in a general time dependent background where "string creation" should be considered in the context of second quantization.

Gravitational plane waves are a particular case of (1) in which $\mathrm{F}$ is quadratic in $X^{a}$, i.e. $F\left(U, X^{a}\right)=W_{a b}(U) X^{a} X^{b}$, and the only non-vanishing component of the Ricci tensor is $R_{U U}=-W_{a}^{a}$. In the so called exact plane waves, $W_{a b}$ is an antisymmetric matrix and thus the metric is Ricci flat. For our purposes in this section it is convenient to restrict attention to a function $W_{a b}$ non-vanishing only in a finite range of U, i.e. $W_{a b}(U) \neq 0$ for $0 \leq U \leq U_{0}$. In this way asymptotic "in" and "out" string states can be defined in the flat space-time regions $U<0$ and $U>U_{0}$.

The action of the bosonic string moving in a plane fronted wave background is

$$
S=\frac{1}{2} \int d^{2} \sigma\left(-\partial_{\alpha} U \partial^{\alpha} V+\partial_{\alpha} X^{a} \partial^{\alpha} X_{a}+F\left(U, X^{a}\right) \partial_{\alpha} U \partial^{\alpha} U\right)
$$

(with $\alpha^{\prime}=1 / 2 \pi$ ).

The classical equations of motion for the transverse fields $X^{a}$ are

$$
\partial_{\alpha} \partial^{\alpha} X_{a}+\frac{1}{2} \partial_{a} F P^{2}=0
$$

where the light cone gauge, $U=P \tau$, was chosen. Transverse coordinates solving (3) automatically satisfy the constraint equations $T_{a b}=0$.

Without loss of generality we assume the background space to be a tensor product of a 4 dimensional plane wave with a $\mathrm{c}=22$ conformal field theory. In the case of exact plane waves with $W_{x x}(U)=-W_{y y}(U)=W_{0}=$ cons. for $0 \leq U \leq U_{0}$, decomposing the two transverse coordinates into modes as

$$
\begin{aligned}
& X(\sigma, \tau)=\sum_{n} X_{n}(\tau) e^{i n \sigma} \\
& Y(\sigma, \tau)=\sum_{n} Y_{n}(\tau) e^{i n \sigma}
\end{aligned}
$$

equation (3) decouples and reads, for each coordinate,

$$
\begin{gathered}
\ddot{X}_{n}+n^{2} X_{n}-W_{0} P^{2} X_{n}=0 \\
\ddot{Y}_{n}+n^{2} Y_{n}+W_{0} P^{2} Y_{n}=0
\end{gathered}
$$

In terms of right and left oscillators, each mode can be written as

$$
X_{n}=\frac{i}{2 \sqrt{n}}\left(a_{n}^{x} u_{n}-\tilde{a}_{n}^{x \dagger} \tilde{u}_{n}\right)
$$

where $u_{n}\left(\tilde{u}_{n}\right)$ are solutions of eq. (6) for $U<0$, i.e. "in" modes of the form $e^{-i n \tau}\left(e^{i n \tau}\right)$. Similarly, "out" modes $v_{n}\left(\tilde{v}_{n}\right)$ can be used for $U>U_{0}$ with the corresponding $b_{n}^{x}, \tilde{b}_{n}^{x}$ oscillators.

A linear Bogoliubov transformation relates "in" and "out" oscillators

$$
\begin{gathered}
b_{n}^{x}=A_{n} a_{n}^{x}-B_{n}^{*} \tilde{a}_{n}^{x \dagger} \\
\tilde{b}_{n}^{x}=A_{n} \tilde{a}_{n}^{x}-B_{n}^{*} a_{n}^{x \dagger}
\end{gathered}
$$

(The oscillators for $Y_{n}$ are obtained from those for $X_{n}$ changing $W_{0} \rightarrow-W_{0}$ ).

The expectation value of the "out" number operator of the $n^{\text {th }}$ right and left modes of a string that was initially in the ground state can be found from these transformations as

$$
\left\langle 0_{\text {in }}\left|N_{n}^{x \quad \text { out }}\right| 0_{\text {in }}\right\rangle=\left\langle 0_{\text {in }}\left|b_{n}^{x \dagger} b_{n}^{x}\right| 0_{\text {in }}\right\rangle=\left|B_{n}^{x}\right|^{2}
$$


This can be used to compute the expectation value of the "out" mass squared operator in the "in" region. Since

$$
M_{\text {out }}^{2}=4 \sum_{n=1}^{\infty} n\left(b_{n}^{a \dagger} b_{n}^{a}+\tilde{b}_{n}^{a \dagger} \tilde{b}_{n}^{a}\right)-8
$$

then

$$
\left\langle M_{\text {out }}^{2}\right\rangle=4 \sum_{n, a} n\left\langle N_{n}^{a}\right\rangle-8
$$

The convergence of this series was used in 77 as a criterion to decide whether a solution is singular or not in the sense of string theory.

The string analogue of the notion of singularity in general relativity is an important question which has already been raised in the literature. Orbifolds are examples of geodesically incomplete geometries where the first quantized string is well defined. However, examples in the opposite direction have also been found. Horowitz and Steif 6 showed that a string trying to propagate through certain singular plane fronted waves becomes infinitely excited. Moreover, since the string can also couple to antisymmetric tensor fields, adding a discontinuous axion to a non singular gravitational background leads to a divergent string state. As a consequence the concept of singularity in string theory is naturally associated to the divergence of the expectation values of physical observables. Here we discuss new examples of divergences in $\left\langle M^{2}\right\rangle$ for geodesically complete metrics.

A careful analysis is needed for divergent profiles. It would be interesting to consider functions $\mathrm{W}(\mathrm{U})$ parametrized in such a way that the limit $W(U) \rightarrow \delta(U)$ can be unambigously taken. The Bogoliubov coefficient $B_{n}$ can be exactly computed for the profile considered above (namely, $\mathrm{W}(\mathrm{U})=W_{0}$ for $0 \leq U \leq U_{0}$ and $\mathrm{W}(\mathrm{U})=0$ otherwise). It turns out to be

$$
\left|B_{n}^{x}\right|^{2}=\left(\frac{1}{2} \frac{P^{2} W_{0}}{n n_{-}} \sin \left(n_{-} U_{0}\right)\right)^{2}
$$

with $n_{ \pm}=\sqrt{n^{2} \pm W_{0} P^{2}}$. Note that the WKB approximation performed in [7] can not be used in this case. Therefore

$$
\left\langle M^{2}\right\rangle=4 \sum_{n=1}^{\infty} \frac{1}{n}\left[\left(\frac{1}{2} \frac{P^{2} W_{0}}{n_{-}} \sin \left(n_{-} U_{0}\right)\right)^{2}+\left(\frac{1}{2} \frac{P^{2} W_{0}}{n_{+}} \sin \left(n_{+} U_{0}\right)\right)^{2}\right]-8
$$

Each of the oscillatory modes of the string gets excited as it passes through the gravitational wave but the expectation value of the mass squared operator remains finite, i.e. the sum in (15) is convergent for $W_{0}$ finite. Some interesting limits can be taken. When $U_{0} \rightarrow 0,\left\langle M^{2}\right\rangle \rightarrow-8$, which is the tachyon mass, the state of the string before the gravitational wave reached it. If $W_{0}$ increases while $U_{0}$ decreases $\left(W_{0} \rightarrow \infty, U_{0} \rightarrow 0\right)$, keeping $W_{0} U_{0}=1$, then the profile $\mathrm{W}(\mathrm{U})$ tends to a delta function $(\mathrm{W}(\mathrm{U}) \rightarrow \delta(U))$ and the expectation value of the number operator yields,

$$
\left\langle N_{n}^{\text {out }}\right\rangle \rightarrow \frac{1}{2}\left(\frac{P^{2}}{n}\right)^{2}
$$

Therefore the mass squared operator $\left\langle M_{\text {out }}^{2}\right\rangle=2 P^{4} \sum_{n=1}^{\infty} \frac{1}{n}-8$ diverges and the string gets infinitely excited. This result can be interpreted either as an indication that the string cannot propagate through the deltiform wave or as a sign that the string solution is unstable (these conclusions neglect possible backreaction effects). As mentioned in [7], the stability of the singularities cannot be analyzed using the singularity theorems of Hawking and Penrose since they do not apply to this case.

Let us now consider a more complicated (continuum) profile: $W(U)=\frac{W_{0}}{\cosh ^{2}\left(\frac{\alpha U}{P}\right)}$. In this case the classical equations of motion (3) read

$$
\ddot{X}_{n}-\frac{W_{0} P^{2}}{\cosh ^{2}(\alpha \tau)} X_{n}=-n^{2} X_{n}
$$

and this can be solved exactly in terms of a hypergeometric function $F(a, b, c ; z)$,

$$
X_{n}(\tau)=(1-\xi)^{-\frac{i n}{2 \alpha}} F\left(-\frac{i n}{\alpha}-s,-\frac{i n}{\alpha}+s+1,-\frac{i n}{\alpha}+1 ; \frac{1+\xi}{2}\right)
$$

where $\xi=\tanh (\alpha \tau)$ and $s=\frac{1}{2}\left(-1+\sqrt{1-\frac{4 W_{0} P^{2}}{\alpha^{2}}}\right)$. The asymptotic expansion of (18) for $\tau \rightarrow \infty$ is, 


$$
X_{n}(\tau) \rightarrow \frac{\Gamma\left(\frac{i n}{\alpha}\right) \Gamma\left(1-\frac{i n}{\alpha}\right)}{\Gamma(-s) \Gamma(1+s)} e^{i n \tau}+\frac{\Gamma\left(-\frac{i n}{\alpha}\right) \Gamma\left(1-\frac{i n}{\alpha}\right)}{\Gamma\left(-\frac{i n}{\alpha}-s\right) \Gamma\left(-\frac{i n}{\alpha}+s+1\right)} e^{-i n \tau}
$$

The Bogoliubov coefficient $B_{n}$ can be easily read from the expression above since it is the coefficient of the negative frequency solution at late times $e^{i n \tau}$, i.e.

$$
\left|B_{n}^{x}\right|^{2}=\left|\frac{\Gamma\left(\frac{i n}{\alpha}\right) \Gamma\left(1-\frac{i n}{\alpha}\right)}{\Gamma(-s) \Gamma(1+s)}\right|^{2}=\left(\frac{\cos \left(\frac{\pi}{2} \sqrt{1-\frac{4 W_{0} P^{2}}{\alpha^{2}}}\right)}{\sinh \left(\frac{\pi n}{\alpha}\right)}\right)^{2}
$$

and $\left|B_{n}^{y}\right|$ is the same expression changing $W_{0} \rightarrow-W_{0}$. In this case the sum in $\left\langle M^{2}\right\rangle$ is always convergent for $W_{0}$ and $\alpha$ finite. Taking $W_{0} \rightarrow \infty, \alpha \rightarrow \infty$ while keeping $\frac{4 W_{0} P^{2}}{\alpha^{2}}=1$ leads to a divergence in the expectation value of the number and square mass operators. This provides another example of a singularity in the sense of string theory whereas space-time is non singular in the sense of general relativity. If the same limits are taken but now keeping $W_{0}=\frac{\alpha}{2}$, then $W(U) \rightarrow \delta(U)$ and we recover the result of eq.(16).

Notice that this is a quantum effect. Classically the masses of the states remain finite since the oscillators are decoupled (eqs.(6), (7)). In the next section we consider the scattering of tachyons in these metrics. Physical amplitudes provide further evidence of the structure of the theory, the spectrum and its symmetries in nontrivial backgrounds.

\section{Tachyon scattering and string spectrum}

The set of coordinates used in the preceeding section $\left(\mathrm{U}, \mathrm{V}, X^{a}\right)$, called harmonic coordinates, is physically convenient since a single chart can be used to cover the whole plane wave space-time and the curvature depends only on one component of the metric tensor. However in order to fully display the symmetries of the geometry and for computational convenience, the so called "group coordinates" are more suitable. In these coordinates the metric takes the form

$$
d s^{2}=-d u d v+g_{a b}(u) d x^{a} d x^{b}
$$

Both sets of coordinates are related by

$$
\begin{aligned}
U & =u \\
V & =v+\frac{1}{2} \dot{g}_{a b}(u) x^{a} x^{b} \\
X^{a} & =P_{b}^{a}(u) x^{b}
\end{aligned}
$$

where $g_{a b}(u)=P_{a}^{c}(u) P_{b}^{c}(u)$ and the matrix $P_{b}^{a}(u)$ is determined by

$$
\ddot{P}_{b}^{a}=W_{a c} P_{b}^{c}(u)
$$

which must be solved with the initial conditions

$$
\dot{P}_{a}^{c}(u) P_{b}^{c}-\dot{P}_{b}^{c} P_{a}^{c}=0
$$

A possible solution is

$$
\begin{aligned}
& P_{1}^{1}(u)=p_{1}(u)=e^{\sqrt{W_{0}} u} \\
& P_{2}^{2}(u)=p_{2}(u)=e^{i \sqrt{W_{0}} u} \\
& P_{2}^{1}(u)=P_{1}^{2}=0
\end{aligned}
$$

The constraint (24) is automatically satisfied by the exact plane waves considered in the previous section. Note that with this choice the metric and coordinates are complex and thus unphysical. Therefore we will consider physical operators those expressed in harmonic coordinates.

In order to compute scattering amplitudes of the lowest energy states in this metric, the vertex operator responsible for the emission of a tachyon is needed. As is well known these vertices must be conformal operators of anomalous dimension 2 and the conditions they must satisfy in an arbitrary curved background were given by Callan and Gan [10. To first order in an $\alpha^{\prime}$ expansion, the tachyon vertex $\mathcal{V}_{T}$ must be a solution of a Klein-Gordon equation

$$
\alpha^{\prime} \Delta \mathcal{V}_{T}^{(p . w .)}\left(k, u, v, x^{a}\right)=k^{2} \mathcal{V}_{T}^{(p . w .)}\left(k, u, v, x^{a}\right)
$$


where we take $\Delta$ as the laplacian in the plane wave (p.w.) metric (21) with $k^{2}=-m^{2}=8$ the mass of the tachyon.

Garriga and Verdaguer 11] found a solution that in this metric reads

$$
\mathcal{V}_{T}^{(p . w .)}\left(k, u, v, x^{a}\right)=: \exp i\left(k_{a} x^{a}-k_{-} v-\frac{1}{4 k_{-}} \int_{0}^{u} d u\left(g^{a b} k_{a} k_{b}+m^{2}\right)+\frac{i}{2} \sqrt{W_{0}}(1+i) u\right):
$$

where $k_{a}, k_{-}$are the separation constants and play the role of components of the momentum of the tachyon. It reduces to the usual vertex operator in flat space when the limit $W_{0} \rightarrow 0$ is taken:

$$
\mathcal{V}_{T}^{(p . w .)} \rightarrow \exp i\left(k_{a} x^{a}-k_{-} v-k_{+} u\right):
$$

It is useful to define a shifted momentum in the direction of $u$ as

$$
\tilde{k}_{+}(u)=\frac{1}{4 k_{-}}\left(g^{a b}(u) k_{a} k_{b}+m^{2}\right)-\frac{i}{2} \sqrt{W_{0}}(1+i)
$$

The four tachyon scattering amplitude on the sphere is defined through

$$
\mathcal{A}_{4 T}=\int \prod_{i=1}^{4} d^{2} z_{i} \int \mathcal{D} u \mathcal{D} v \mathcal{D} x^{a}|J| \prod_{i=1}^{4} \mathcal{V}_{T}^{(p . w .)}\left(k_{i}, z_{i}\right) e^{i S[u, v, x]}
$$

where the vertex $\mathcal{V}_{T}^{(p . w .)}\left(k_{i}, z_{i}\right)$ is placed at the point $z_{i}$ of the complex plane; $|J|$ is the determinant of the jacobian of the transformation between harmonic and group coordinates (it is included because the physical vertices are assumed to be those in harmonic coordinates, but we shall see that it does not contribute to the amplitude due to momentum conservation) and $S[u, v, x]$ is the action expressed in group coordinates:

$$
S\left[u, v, x^{a}\right]=\frac{1}{2} \int d^{2} z\left(-\partial_{\alpha} u \partial^{\alpha} v+g_{a b}(u) \partial_{\alpha} x^{a} \partial^{\alpha} x^{b}\right)
$$

It is possible to simplify the calculations by making a Lorentz transformation so that all $k_{-}^{(i)}$ vanish, except two of them. We then consider

$$
\begin{array}{ll}
k_{-}^{(i)}=0, & i=2,3 \\
k_{-}^{(i)} \neq 0, & i=1,4
\end{array}
$$

Of course this transformation is not a symmetry of the metric. However this loss of generality is irrelevant for our purposes, as we shall see. With this choice the vertices $\mathcal{V}_{2}^{(p . w .)}$ and $\mathcal{V}_{3}^{(p . w .)}$ have the same form as the Minkowski space vertices (with $k_{-}=0$ ), because equation (26) only depends on the transverse coordinates. This independence of $v$ means that these two tachyons travel in the same direction as the gravitational wave and never collide with it:

$$
\mathcal{V}_{2,3}=e^{-i k_{+} u+i k_{a} x^{a}}
$$

Putting everything together in the functional integral, the amplitude $\mathcal{A}_{4 T}$ in eq (30) reads:

$$
\begin{aligned}
\mathcal{A}_{4 T}=\int \mathcal{D} u \mathcal{D} v \mathcal{D} x^{a}|J| & \exp i\left[\sum_{i=1}^{4} k_{a}^{(i)} x^{a}\left(z_{i}\right)-\sum_{i=1,4} k_{-}^{(i)} v\left(z_{i}\right)\right. \\
& \left.-\sum_{i=1}^{4} \int_{0}^{u} d u \tilde{k}_{+}^{(i)}\left(u\left(z_{i}\right)\right)-\sum_{i=2,3} k_{+}^{(i)} u\left(z_{i}\right)\right] \times e^{i S[u, v, x]}
\end{aligned}
$$

The jacobian $J\left(\frac{\partial X^{\mu}}{\partial x^{\nu}}\right)$ can be easily evaluated because it depends only on $u$, then

$$
\operatorname{det} J=\prod_{z, \bar{z}} p_{1}(u) p_{2}(u)=\exp \left(\sqrt{W_{0}}(1+i) \int d^{2} z u(z)\right)
$$

Now integrating the action by parts and collecting all the pieces under the same integral symbol in the exponential by introducing delta functions $\delta^{2}\left(z-z_{a}\right), \mathcal{A}_{4 T}$ can be expressed as

$$
\mathcal{A}_{4 T}=\int \prod_{i=1}^{4} d^{2} z_{i} \int \mathcal{D} u \mathcal{D} v \mathcal{D} x^{a} e^{\tilde{S}\left[u, v, x^{a}, k_{a}^{(i)}, z_{i}\right]}
$$


where

$$
\begin{aligned}
\tilde{S}=\int d^{2} z[ & v\left(\frac{1}{2} \partial_{\alpha} \partial^{\alpha} u-\sum_{i=1,4} k_{-}^{(i)} \delta^{2}\left(z-z_{i}\right)\right)-\sum_{i=2,3} k_{+}^{(i)} u\left(z_{i}\right) \delta^{2}\left(z-z_{i}\right)+ \\
& +\int_{0}^{u(z)} d u\left(\sqrt{W_{0}}(1+i)-\sum_{i=1,4} \tilde{k}_{+}^{(i)}(u) \delta^{2}\left(z-z_{i}\right)\right) \\
& \left.+x^{a}\left(-\frac{1}{2} \partial_{\alpha} g_{a b} \partial^{\alpha}\right) x^{b}+\sum_{i=1}^{4} k_{a}^{(i)} x^{a} \delta^{2}\left(z-z_{i}\right)\right]
\end{aligned}
$$

Due to the linear dependence the integral on $v$ can be performed, giving the following delta function

$$
\delta\left[\frac{1}{2} \partial_{\alpha} \partial^{\alpha} u-\sum_{i=1,4} k_{-}^{(i)} \delta^{2}\left(z-z_{i}\right)\right]
$$

Integrating $u$ therefore simply implies replacing it by

$$
\bar{u}(z)=\sum_{i=1,4} k_{-}^{(i)} \ln \left|z-z_{i}\right|
$$

and inserting the factor $\operatorname{det}^{-1}\left(\partial_{\alpha} \partial^{\alpha}\right)$, irrelevant for our calculations.

We are then left with

$$
\begin{aligned}
\mathcal{A}_{4 T} \sim & \int \prod_{i=1}^{4} d^{2} z_{i} \exp \int d^{2} z \int_{0}^{\bar{u}(z)} d u\left[-\sum_{i=1,4} \tilde{k}_{+}^{(i)} \delta^{2}\left(z-z_{i}\right)-\sum_{i=2,3} k_{+}^{(i)} \delta^{2}\left(z-z_{i}\right)\right] \\
& \times \int \mathcal{D} x^{a} \exp \int d^{2} z\left[x^{a}\left(-\frac{1}{2} \partial_{\alpha} g_{a b} \partial^{\alpha}\right) x^{b}+\sum_{i=i}^{4} k_{a}^{(i)} x^{a}(z) \delta^{2}\left(z-z_{i}\right)\right]
\end{aligned}
$$

Note that the contribution from the Jacobian vanishes due to momentum conservation. Finally, one can integrate over $x^{a}$ since the action is quadratic in those fields. This yields for the amplitude

$$
\begin{gathered}
\mathcal{A}_{4 T} \sim \int \prod_{i=1}^{4} d^{2} z_{i} \exp \left[-k_{+}^{(2)} \bar{u}\left(z_{2}\right)-k_{+}^{(3)} \bar{u}\left(z_{3}\right)-\int_{0}^{\bar{u}\left(z_{1}\right)} d u \tilde{k}_{+}^{(1)}-\int_{0}^{\bar{u}\left(z_{4}\right)} d u \tilde{k}_{+}^{(4)}\right] \\
\times \exp \left[\frac{1}{4} \int d^{2} z d^{2} z^{\prime} \sum_{i=1}^{4} k_{a}^{(i)} \delta^{2}\left(z-z_{i}\right) A^{a b}\left(z, z^{\prime}\right) \sum_{j=i}^{4} k_{b}^{(j)} \delta^{2}\left(z^{\prime}-z_{j}\right)\right]
\end{gathered}
$$

where $A^{a b}\left(z, z^{\prime}\right)$ is the Green function of the quadratic operator $-\frac{1}{2} \partial_{\alpha} g_{a b} \partial^{\alpha}$,i.e.:

$$
-\frac{1}{2} \partial_{\alpha} g_{a b}(\bar{u}(z)) \partial^{\alpha} A^{a b}\left(z, z^{\prime}\right)=\delta^{2}\left(z, z^{\prime}\right)
$$

Using expression (38) for $\bar{u}(z)$, we may write

$$
\begin{aligned}
& \mathcal{A}_{4 T} \sim \int \prod_{i=1}^{4} d^{2} z_{i}\left|z_{2}-z_{1}\right|^{-k_{+}^{(2)} k_{-}^{(1)}}\left|z_{2}-z_{4}\right|^{-k_{+}^{(2)} k_{-}^{(4)}}\left|z_{3}-z_{1}\right|^{-k_{+}^{(3)} k_{-}^{(1)}}\left|z_{3}-z_{4}\right|^{-k_{+}^{(3)} k_{-}^{(4)}} \\
& \times \exp \left[-\int_{0}^{\bar{u}\left(z_{1}\right)} d u \tilde{k}_{+}^{(1)}-\int_{0}^{\bar{u}\left(z_{4}\right)} d u \tilde{k}_{+}^{(4)}\right] \\
& \times \exp \left[\frac{1}{2} \sum_{i<j} k_{a}^{(i)} k_{b}^{(j)} A^{a b}\left(z_{i}, z_{j}\right)\right]
\end{aligned}
$$

Since the vertices are normal ordered, selfcontractions are not considered. Note that the shift in $\tilde{k}_{+}^{(1)}$ and $\tilde{k}_{+}^{(4)}$ can be dropped in this case because of momentum conservation in $k_{-}$, (i.e, $\left.\left[\bar{u}\left(z_{1}\right)+\bar{u}\left(z_{4}\right)\right]=0\right)$, then we can replace $\int_{0}^{\bar{u}} d u \tilde{k}_{+}^{(i)} \rightarrow \int_{0}^{\bar{u}} d u k_{+}^{(i)}$. Therefore nontrivial interactions take place in the transverse space. 
The next step is to compute the Green function (41) writing the operator $-\frac{1}{2} \partial_{\alpha} g_{a b} \partial^{\alpha}$ in the form

$$
\Delta(z)=-\frac{1}{2} \partial_{\alpha} g_{a b} \partial^{\alpha}=-p^{2}\left(\Delta_{0}+\delta \Delta\right)
$$

where

$$
\begin{aligned}
\Delta_{0} & =\frac{1}{2} \partial_{\alpha} \partial^{\alpha}=2 \partial_{z} \bar{\partial}_{\bar{z}} \\
\delta \Delta & =\partial_{z} \ln p^{2} \bar{\partial}_{\bar{z}}+\bar{\partial}_{\bar{z}} \ln p^{2} \partial_{z}
\end{aligned}
$$

and $p$ is either $p_{1}(\bar{u})$ or $p_{2}(\bar{u})$. Note that $\delta \Delta$ is proportional to $\sqrt{W_{0}}$, so if we consider $\delta \Delta$ as a perturbation to the plane Green function $\Delta_{0}$, formally,

$$
\begin{aligned}
& \Delta(z)=-p^{2} \Delta_{0}\left[1+\int d^{2} \omega \Delta_{0}^{-1}(z-\omega) \delta \Delta(\omega)\right] \\
& \Delta_{0}^{-1}(z-\omega)=-2 \ln |z-\omega|
\end{aligned}
$$

Then, to first order in $\sqrt{W_{0}}$ :

$$
\Delta^{-1}\left(z, z^{\prime}\right) \approx-\Delta_{0}^{-1}\left(z-z^{\prime}\right) p^{-2}\left(z^{\prime}\right)+p^{-2}\left(z^{\prime}\right) \int d^{2} \omega \Delta_{0}^{-1}(z-\omega) \delta \Delta(\omega) \Delta_{0}^{-1}\left(\omega-z^{\prime}\right)
$$

Therefore $A^{a b}\left(z, z^{\prime}\right) \approx \Delta^{-1}\left(z, z^{\prime}\right)$, with $p^{2}=p_{1}^{2}(\bar{u})$ for $a=b=1$ and $p^{2}=p_{2}^{2}(\bar{u})$ for $a=b=2$. Of course in the limit $W_{0} \rightarrow 0$ we recover the plane Green function.

We now discuss the factorization of this amplitude $\left(\mathcal{A}_{4 T}\right)$ when two of the external vertices are placed at the same point. In this way the mass spectrum of the theory can be found from the physical poles corresponding to the particles interchanged in the process. The residues give rise to the scattering amplitude of the intermediate state with the remaining tachyons. From them, applying the procedure introduced in reference [12], the vertex operators of the exchanged states can be read.

An $N$-tachyon amplitude $\mathcal{A}_{N}\left(k^{(1)}, \ldots, k^{(N)}\right)$, factorizes when $r$ vertices collide to the same point, i.e. taking the limit $z_{i} \rightarrow z_{r}$ for $i=1, \ldots, r-1$, as

$$
\mathcal{A}_{N} \longrightarrow \frac{1}{\frac{1}{4} K^{2}-2} \mathcal{A}_{r+1}\left(k^{(1)}, \ldots, k^{(r)},-K\right) . \mathcal{A}_{N-r+1}\left(K, k^{(r+1)}, \ldots, k^{(N)}\right)
$$

where $K_{\mu}=\sum_{i=1}^{r} k_{\mu}^{(i)}$ is the momentum of the intermediate state, and the pole occurs at the physical mass $m^{2}=-8$, i.e. the particle exchanged is a tachyon. Taylor expanding the residue, new poles corresponding to higher mass intermediate particles are found.

In fact, given a function $\Phi(\epsilon, \bar{\epsilon})$, regular for $\epsilon, \bar{\epsilon} \rightarrow 0$, a Taylor expansion leads to

$$
\begin{aligned}
\int d^{2} \epsilon|\epsilon|^{-2 \nu} \Phi(\epsilon, \bar{\epsilon}) & =\left.\int d^{2} \epsilon|\epsilon|^{-2 \nu} \sum_{l, m} \frac{\epsilon^{l} \bar{\epsilon}^{m}}{l ! m !} \partial^{l} \bar{\partial}^{m} \Phi\right|_{0} \quad \epsilon^{l} \bar{\epsilon}^{m} \\
& =\left.\sum_{n} \frac{\Lambda^{-2 \nu+2 n+2}}{-2 \nu+2 n+2} \frac{1}{(n !)^{2}}(\partial \bar{\partial})^{n} \Phi\right|_{0}
\end{aligned}
$$

where angular integration in polar coordinates implies $l=m=n$ and $\Lambda$ is an arbitrary cutoff, irrelevant for $\nu \rightarrow n+1$.

Taking the limit $z_{1} \rightarrow z_{2},\left(\epsilon=z_{1}-z_{2}\right)$ from the amplitude $(42)$, the momenta of the colliding tachyons are $k_{-}^{(1)} \neq 0$ and $k_{-}^{(2)}=0$ so that the momentum of the intermediate state $K_{\mu}$ is general, i.e. $k_{-}^{(1)}+k_{-}^{(2)} \neq 0$. It is possible to isolate the divergent part of the amplitude (replacing $z_{1}$ by $z_{2}+\epsilon$ ), as

$$
\begin{aligned}
& \mathcal{A}_{4 T} \sim \prod_{i=2}^{4} \int d^{2} z_{i} \int d^{2} \epsilon|\epsilon|^{\frac{1}{2} k^{(2)} \cdot k^{(1)}}\left|z_{23}+\epsilon\right|^{-k_{+}^{(3)} \cdot k_{-}^{(1)}}\left|z_{24}\right|^{-k_{+}^{(2)} \cdot k_{-}^{(4)}}\left|z_{34}\right|^{-k_{+}^{(3)} \cdot k_{-}^{(4)}} \\
& \times \exp \left(-\int_{0}^{\bar{u}\left(z_{2}+\epsilon ; \epsilon\right)} d u \tilde{k}_{+}^{(1)}(u)-\int_{0}^{\bar{u}\left(z_{4} ; \epsilon\right)} d u \tilde{k}_{+}^{(4)}\right) \\
& \times \exp \left(\frac{1}{2} \sum_{j>2} k_{a}^{(1)} \cdot k_{b}^{(j)} A^{a b}\left(z_{2}+\epsilon ; z_{j} ; \epsilon\right)+\frac{1}{2} \sum_{2<i<j} k_{a}^{(i)} k_{b}^{(j)} A^{a b}\left(z_{i}, z_{j} ; \epsilon\right)\right)
\end{aligned}
$$


and therefore identify $\nu=-\frac{1}{4} k^{(1)} \cdot k^{(2)}$. Integrating $\epsilon$ after Taylor expanding the regular part the poles at $\nu=n+1$ correspond to $K^{2}=\left(k^{(1)}+k^{(2)}\right)^{2}=-8(n-1)$, i.e. the interaction with the gravitational wave does not change the mass spectrum of the theory with respect to the Minkowskian case. This need not be true in presence of a nontrivial dilaton background. As shown in reference [8] in this case the graviton acquires a (tachyonic) mass proportional to the derivative of the dilaton.

We now turn to analize the residues. For $n=0$ we find the tachyon pole $\left(k^{(1)} \cdot k^{(2)}=-4\right.$ or equivalently $\left(k^{(1)}+\right.$ $\left.k^{(2)}\right)^{2}=-m^{2}=8$ ) and the residue corresponds to the product of two 3-tachyon amplitudes, (one of them is already divided by the (infinite) volume of the conformal group, leaving only a constant as a result).

\section{The graviton vertex operator}

For $n=1$, i.e. the massless pole $K^{2}=0$, the derivatives $\partial_{\epsilon} \bar{\partial}_{\epsilon} \Phi(\epsilon, \bar{\epsilon})$ lead to a residue corresponding to the scattering of a massless vertex with two tachyons. We find

$$
\begin{aligned}
\mathcal{A}_{G T T}^{(p . w .)} & =\mid \frac{1}{2} \frac{k_{+}^{(3)} k_{-}^{(4)}}{z_{23}}+\frac{1}{2} \frac{\tilde{k}_{+}^{(1)}\left(\bar{u}\left(z_{2}\right)\right) k_{-}^{(4)}}{z_{24}}+\frac{1}{2} \frac{\tilde{k}_{+}^{(4)}\left(\bar{u}\left(z_{4}\right)\right) k_{-}^{(1)}}{z_{24}}+ \\
& +\frac{1}{2} \sum_{i=3}^{4} k_{a}^{(1)} k_{b}^{(i)}\left[\partial_{2} A^{a b}\left(z_{i}, z_{2}\right)+\partial_{2} A^{a b}\left(z_{2}, z_{i}\right)\right] \\
& -\left.\frac{1}{2} \sqrt{W_{0}} k_{-}^{(1)} k_{a}^{(3)} k_{b}^{(4)}\left[\frac{A^{a b}\left(z_{3}, z_{4}\right)}{z_{24}}+\frac{A^{a b}\left(z_{4}, z_{3}\right)}{z_{23}}\right]\right|^{2} \times \mathcal{A}_{3 T}\left(K, k^{(3)}, k^{(4)}\right)
\end{aligned}
$$

where $\mathcal{A}_{3 T}$ is the 3 -tachyon amplitude for states of momentum $K_{\mu}=k_{\mu}^{(1)}+k_{\mu}^{(2)}, k_{\mu}^{(3)}, k_{\mu}^{(4)}$.

Note that the shifts in $\tilde{k}_{+}^{(1)}$ and $\tilde{k}_{+}^{(4)}\left(\tilde{k}_{+}=k_{+}-\frac{i}{2} \sqrt{W_{0}}(1+i)\right)$ decouple from the physical processes if momentum conservation $k_{-}^{(1)}+k_{-}^{(4)}=0$ is used (recall equation (42)). However this is a consequence of the particular choice $k_{-}^{(2)}=k_{-}^{(3)}=0$ we made, and in general this will not happen. The Minkowskian limit $\mathcal{A}_{G T T}^{(M)}$ is recovered when $W_{0} \rightarrow 0$.

Since the information on the plane wave background is contained in the contribution from the transverse coordinates and in the shift in $k_{+}$, when the transverse momentum of the particles vanish, $\mathcal{A}_{G T T}^{(p . w .)}$ reduces to $\mathcal{A}_{G T T}^{(M)}$ if conservation of $k_{-}$is used. This means that the particles collide normally to the gravitational wave and they do not "feel" the background. Nontrivial interactions take place in the transverse space. On the other hand if all the $k_{-}$components vanish, again $\mathcal{A}_{G T T}^{(M)}$ is recovered.

From this amplitude it is possible to obtain the massless vertex operator. A generic operator of naive conformal dimension 2 on a flat world sheet is of the form $\partial_{\alpha} X^{\mu} \partial^{\alpha} X^{\nu} F_{\mu \nu}(X)$. Translation invariance implies an exponential of the same form as the tachyon vertices. With these considerations we can read from eq. (52) the operator responsible for the emission or absorption of a massless state as

$$
\mathcal{V}_{G}^{(p . w .)}=\tilde{\epsilon}_{\mu \nu}(k): \partial X^{\mu} \bar{\partial} X^{\nu} e^{-i K_{-} v+i K_{i} X^{i}-\frac{1}{4 k_{-}} \int_{0}^{u} d u \tilde{k}_{+}(u)}:
$$

with $X^{\mu}=\left(u, v, X^{1}, X^{2}\right), \tilde{\epsilon}_{\mu \nu}=\tilde{\epsilon}_{\mu} \cdot \tilde{\epsilon}_{\nu} ; \quad \tilde{\epsilon}_{\mu}=\epsilon_{\mu}+\frac{i}{2} \sqrt{W_{0}}(1+i) \delta_{\mu}^{u}$ and $\epsilon_{\mu}=k_{\mu}^{(1)}$.

Indeed by computing the scattering amplitude of this vertex operator with two tachyons it can be checked that $\mathcal{A}_{G T T}^{(p . w .)}$ in eq. (52) is recovered. (Recall that $\mathcal{A}_{G T T}^{(p . w .)}$ was obtained by factorizing $\mathcal{A}_{4 T}^{(p . w .)}$ ). The polarization tensors obtained in this way are of course particular ones. They depend only on $k_{\mu}^{(1)}$ because of the particular vertices that were made to coincide and the way the limit $z_{1} \rightarrow z_{2}$ was taken. However, once the conditions to be satisfied by the polarization tensors are imposed, namely transversality $K^{\mu} \epsilon_{\mu \nu}=0$, the form of the vertex operator is completely general. Notice that the polarization tensor can be decomposed into a traceless part (graviton) and a trace part (dilaton). The antisymmetric tensor cannot be produced in this way since it does not couple to tachyons.

Callan and Gan 10] deduced the conditions that a massless vertex must satisfy in order to be an eigenoperator with eigenvalue two of the anomalous dimension matrix in a general $\sigma$-model background. They define a general operator of naive dimension two as

$$
V=F_{\mu \nu} \partial X^{\mu} \bar{\partial} X^{\nu}+\alpha^{\prime(2)} R F(X)
$$

where the second term is unavoidable when studying string theory on a world sheet of curvature ${ }^{(2)} R$. Setting the dilaton to zero on a plane world sheet the equations to be satisfied by the massless vertex operators are

$$
\begin{aligned}
& -\nabla^{2} F_{\mu \nu}-\nabla \mu \nabla_{\nu} F_{\lambda}^{\lambda}+R_{\mu}^{\lambda \sigma}{ }_{\nu} F_{\lambda \sigma}+2 \nabla_{\mu} \nabla^{\lambda} F_{\lambda \nu}+2 \nabla_{\nu} \nabla^{\lambda} F_{\lambda \mu}+\nabla_{\mu} \nabla_{\nu} F=0 \\
& \frac{1}{4} \nabla^{2} F_{\lambda}^{\lambda}-\frac{1}{4} \nabla^{\lambda} \nabla^{\sigma} F_{\lambda \sigma}+\frac{1}{4} R^{\lambda \sigma} F_{\lambda \sigma}-\nabla^{2} F=0
\end{aligned}
$$


Using the Ricci-flat metric (21) whose nonvanishing Christoffel symbols and Riemann tensor reduce to:

$$
\begin{aligned}
& \Gamma_{b u}^{a}=\frac{1}{2} g^{a c} \dot{g}_{c b} ; \quad \Gamma_{a b}^{v}=\dot{g}_{a b} \\
& R_{u b u}^{a}=-\partial_{u} \Gamma_{b u}^{a}-\Gamma_{c u}^{a} \Gamma_{b u}^{c}
\end{aligned}
$$

these equations are satisfied by

$$
F_{\mu \nu}(x)=\tilde{\epsilon}_{\mu \nu} e^{-i K_{-} v+i K_{a} x^{a}-\frac{i}{4 K_{-}} \int_{0}^{u} d u \tilde{K}_{+}}
$$

with $\tilde{\epsilon}_{\mu \nu}$ given by eq.(53). This can be verified using that $K^{2}=0$ and the transversality condition $K_{\mu} \epsilon^{\mu \nu}=0$. Notice that even though the shift in $\tilde{k}_{+}^{(1)}$ can be elliminated from the physical amplitude (52) using momentum conservation, it is unavoidable in order to satisfy equations (55). The function $F(x)$ is a tachyon-like operator with momentum $K_{\mu}$, so $\nabla^{2} F=K^{2} F=0$. Similarly $F_{\mu}^{\mu}=\tilde{\epsilon}_{\mu}^{\mu}=\left(k^{(1)}\right)^{2}+2 i k_{-} \sqrt{W_{0}}(1+i)=-m^{2}+2 i k_{-} \sqrt{W_{0}}(1+i), m$ being the tachyon mass. Then $\nabla^{2} F_{\mu}{ }^{\mu}=\left[-m^{2}+2 i k_{-} \sqrt{W_{0}}(1+i)\right] K^{2} F=0$.

\section{Conclusions}

We considered string theory in plane wave backgrounds. Profiles of the gravitational wave where the Bogoliubov transformation can be exactly solved were found. The mass squared operator remains convergent as long as the 'height' of the waves is finite. The limit of a deltiform wave was unambigously taken, leading to a divergent mass squared operator.

Tachyon scattering amplitudes were constructed and solved to first order in a perturbative expansion in $\sqrt{W_{0}}$. The information about the gravitational wave is contained both in the transverse coordinates and in the shift in $\tilde{k}_{+}$. Two particular situations can be distinguished: a)if all $k_{-}^{(i)}=0$, i.e. none of the tachyons collide with the wave, then the interaction reduces to the flat space case; b) if all transverse momenta vanish, i.e. the particles collide normally to the wave, again the interaction reduces to the Minkowskian case. A similar observation was made in reference 113 where the current algebra giving rise to this class of backgrounds was analyzed. However the gravitational wave obtained from the central extension of the 2D Poincaré algebra 15$]$ is different from the one we have considered here.

From the poles appearing in the factorization of this amplitude the mass spectrum of the theory was found to coincide with the flat space case. The vertex operator of the massless states was 'read' from the residue of the massless pole corresponding to the graviton-tachyon scattering amplitude. The effect of the wave on the vertex is to shift the $k_{+}$momentum component by a factor $\frac{i}{2} \sqrt{W_{0}}(1+i)$. The vertices responsible for the emission or absorption of higher mass particles can be constructed in the same way from higher orders of the Taylor expansion. We believe that the difference with their flat space analogues will be contained in the shift in $\tilde{k}_{+}$and in the exponential "tachyonic" part.

The procedure used here to obtain the higher mass vertex operators can be generalized to arbitrary Riemann surfaces 12 . The advantage of the formalism is mainly that normal ordering and selfcontractions arise naturally from the physical amplitudes. Arbitrary regularizations and possible sources of Weyl anomalies are thus avoided.

It could be interesting to analyze duality in this context.

\section{ACKNOWLEDGMENTS}

We are grateful to J.M. Maldacena for collaboration in the initial stages of this work. This work was partially supported by Consejo Nacional de Investigaciones Científicas y Técnicas (Argentina); the Directorate General for Science, Research and Development of the Commision of the European Communities under contract No. C11-0540M(TT); Universidad de Buenos Aires and Fundación Antorchas

[1] D. Amati and C. Klimcik, Phys. Lett. B219, 443(1989)

[2] D. Amati, M. Ciafaloni and G. Veneziano, Phys. Lett. B197, 81(1987)

[3] K. Kikkawa and M. Yamasaki, Phys. Lett. B149, 357(1984)

[4] W. Siegel, Phys. Lett. B134, 318(1984); B. Fridling and A. Jeviki, Phys. Lett. B134, 70(1984); T. Buscher, Phys. Lett. B194, 59(1987)

[5] G. Horowitz and D. Welch, Phys. Rev. Lett. 71, 328(1993) 
[6] G. Horowitz and R. Steif, Phys. Rev. Lett. 64, 260(1990)

[7] G. Horowitz and R. Steif, Phys. Rev. D 42, 1950(1990)

[8] Antoniadis, C. Bachas, J. Ellis and D. Nanopoulos, Phys. Lett. B211, 393(1988)

[9] R. Penrose, Phys. Rev. Lett. 14, 14(1965);R. Penrose and S. Hawking, Proc. Roy. Soc. Lond. A314, 529(1970)

[10] C. Callan and Z. Gan, Nucl. Phys. B272, 647(1986)

[11] J. Garriga and E. Verdaguer, Phys. Rev. D 43, 391(1991)

[12] G. Aldazabal, M. Bonini, R. Iengo and C. Núñez, Phys. Lett. B199, 41(1987)

[13] E. Kiritsis and C. Kounnas, String Propagation in Gravitational Wave Backgrounds, CERN preprint, hepth/9310202

[14] N. Sánchez and H. de Vega, Phys. Lett. B244, 215(1990); Nucl. Phys. B 317, 706(1989)

[15] C. Nappi and E. Witten, A WZW Model Based on a Non-semisimple Group, IAS preprint, hepth/9310112

[16] E. Witten, Phys. Rev. D 44, 314(1991) 